\title{
Presence of Contaminants in Refrigerant and Their Effect on Refrigeration Cycle
}

\author{
Dorota Skrzyniowska \\ Cracow University of Technology, Cracow 31-155, Poland
}

Received: August 26, 2013 / Accepted: October 09, 2013 / Published: February 28, 2014.

\begin{abstract}
Refrigerant is a medium used in refrigeration cycle. Until now in thermodynamic models calculations of refrigerant is not considered with lubricant and moist air but as an ideal pure gas (without any contaminants). This is also the case in refrigeration cycle where the refrigerant is very often considered as an ideal one. Lubricant, air and water vapour are not considered in thermodynamic calculations in spite of their existence. The problems in the existing research are summarized. A further study is suggested on refrigerant systems. This circulation of such calculations is at the origin of a deviation from a theoretical behaviour. This article aims at reviewing the researches of oil-refrigerant, air-refrigerant, moist air-refrigerant or oil-moist air-refrigerant mixtures and focuses particularly on mixture enthalpy calculation. This paper deals with the description of the real mixture of refrigerant and contaminants (lubricant, water, moist air and other remains of refrigerants at elevated pressures and temperatures) which flow in a refrigerating cycle and the main point of this paper is to appoint thermodynamic parameters of such a mixture using the thermodynamic formulas.
\end{abstract}

Key words: Refrigeration system, compression system, lubrication, oil, modeling, air conditioning, compressed air, thermodynamic, specific enthalpy.

\section{Nomenclature}

$\begin{array}{ll}a_{p i s t} & \text { Acceleration of piston }\left(\mathrm{m} / \mathrm{s}^{2}\right) \\ A_{p} & \text { Cross sectional area of cylinder }\left(\mathrm{m}^{2}\right) \\ D_{i n} & \text { Inside diameter of cylinder }(\mathrm{m}) \\ g & \text { Gravitational acceleration }\left(\mathrm{m} / \mathrm{s}^{2}\right) \\ h & \text { Specific enthalpy }(\mathrm{J} / \mathrm{kg}) \\ \dot{m} & \text { Mass flux }(\mathrm{kg} / \mathrm{s}) \\ O b & \text { Cross-sectional circumference of cylinder }(\mathrm{m}) \\ p, p_{o}, p_{k}, p_{r} & \text { Pressure, inlet pressure, outlet pressure, reduced } \\ \dot{q}_{Z} & \text { pressure }(\mathrm{Pa}) \\ s & \text { Rate of heat flow }\left(\mathrm{W} / \mathrm{m}^{2}\right) \\ T, t & \text { Piston stroke }(\mathrm{m}) \\ v & \text { Temperature } \mathrm{K}\left({ }^{\circ} \mathrm{C}\right) \\ w & \text { Molar volume of the mixture }\left(\mathrm{m}^{3} / \mathrm{kg}\right) \\ \kappa & \text { Velocity }(\mathrm{m} / \mathrm{s}) \\ \tau & \text { Polytrophic exponent }(-) \\ \tau_{0} & \text { Time }(\mathrm{s}) \\ Z & \text { Medium tangential stress on cylinder's wall } \\ & \text { (shear) }\left(\mathrm{kg} / \mathrm{m} \mathrm{s}^{2}\right) \\ & \text { Coordinate along axis of cylinder }(\mathrm{m})\end{array}$

Corresponding author: Dorota Skrzyniowska, Ph.D., research fields: air conditioning, refrigeration, environmental engineering. E-mail: skdorota@pk.edu.pl.

\section{Introduction}

The evaporator, compressor (in author's research-Reciprocating), condenser and expansion valve are essential components of refrigeration cycle. The vapor-compression refrigeration system uses a circulating liquid refrigerant as the medium that absorbs and removes heat from the space to be cooled and subsequently rejects that heat elsewhere.

In order to lubricate the moving parts of the compressor, oil is added. The type of oil may be mineral or synthetic to suit the compressor type, and chosen so as not to react with the refrigerant type and other components in the system. In small refrigeration systems, the oil is allowed to circulate throughout the whole circuit, but care must be taken to design the pipework and components such that oil can drain back under gravity to the compressor.

In larger more distributed systems, especially in retail refrigeration, oil is normally captured at an oil separator immediately after the compressor, and is in 
turn re-delivered, by an oil level management system, back to the compressor(s). Oil separators are not $100 \%$ efficient so system pipework must still be designed so that oil can drain back by gravity to the oil separator or compressor. Presence of lubricant and moisture is accounted for seriously as problems of engineering and environmental protection. Contaminants in refrigeration system influence thermodynamic parameters of the refrigerant. These parameters influence the effectiveness of heat exchange in the heat exchanger and the work of compressor and the throttling valve (expansion valve) $[1,2]$.

The presence of these contaminants should be taken into account in calculations. The incidence of oil, moisture and air induces changes in the flow configurations, pressure drops and properties of the refrigerants such as enthalpy, viscosity, surface tension, etc.. For these reasons, the author tries to analyze the influence of the contaminants on the refrigeration calculations.

\section{The Presence of Air in a Refrigeration Cycle}

Air can get inside a refrigeration system through a couple of ways. Air enters the system when the system is opened for service. Air gets inside installation by leakage (i.e. valve spindle, flange yoke, stuffing box of crankshaft, which are characteristics for refrigeration units working at vapor pressure lower than atmospheric pressure) during exchange as the remainder after insufficiently purification installation. Air can get inside installation at refrigerant make-up.

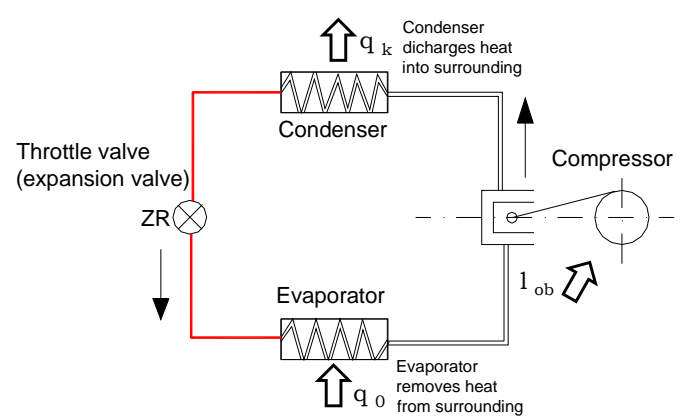

Fig. 1 A typical single-stage vapor compression refrigeration system.
Refrigerant as a trade product can contain up to $5 \%$ of air.

Another way that air often enters the system is during the refrigerant charging process. If the refrigerant tank on a charging station or recycling equipment contains air, it will enter the cycle along with the refrigerant. Some recycling machines are designed to automatically vent trapped air from the recovery tank while others require this job to be done manually. Some technicians are not as meticulous as others, and older equipment does not automatically purge the tanks as well as it should. Consequently, air contaminated refrigerant is a common problem. A properly functioning system with a normal charge of uncontaminated refrigerant should blow air that is about $40{ }^{\circ} \mathrm{C}$ to $50{ }^{\circ} \mathrm{C}$ colder than ambient temperatures. This will obviously vary with temperature and humidity, but should be a ballpark figure.

Air aggregates in high-pressure elements of installation, in places where lowest velocity of vapour refrigerant and temperature occur, which are mainly in condenser and liquid reservoir (in Fig. 2).

Only refrigerant condenses during condensation, whereas air remains gaseous, which aggravates near the heat exchange surface in condenser [3] (even smattering of air around pipe or inside pipe cause several decrease of heat exchange coefficient causing increase of pressure in heat exchanger above saturation pressure) in Fig. 3 [4].

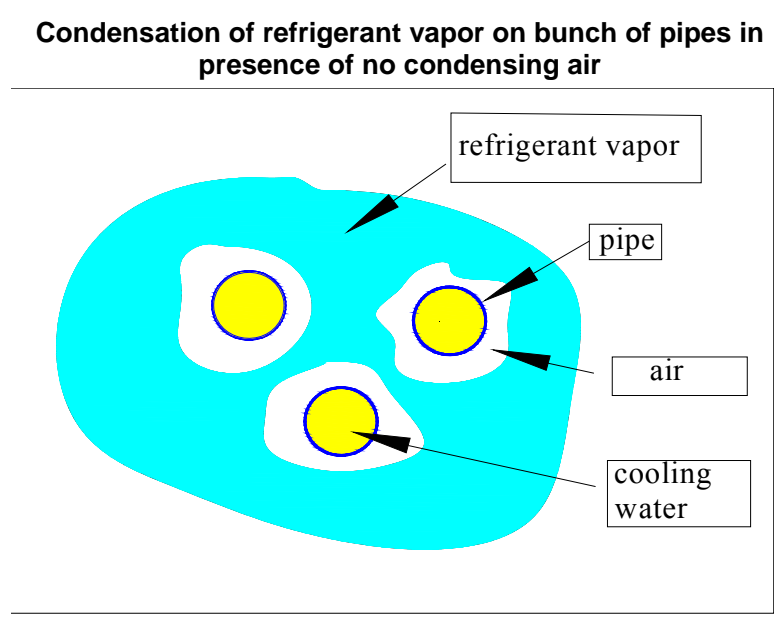

Fig. 2 Configuration of layer a round coil pipe. 


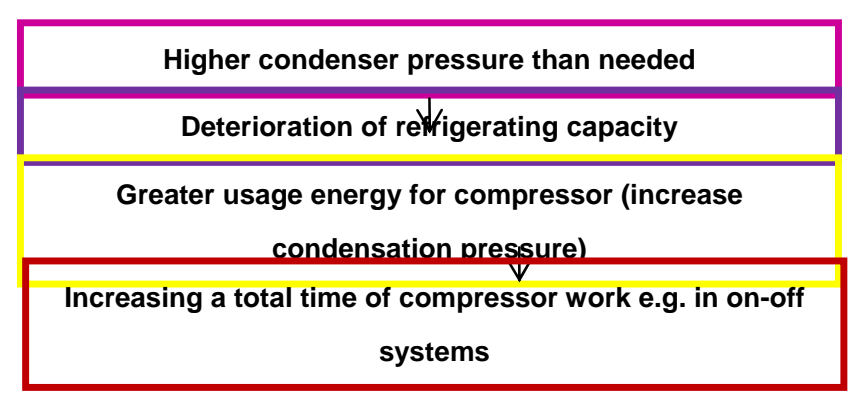

Fig. 3 The effect of inert gases in refrigeration.

Results of inert gases presence in refrigerant cycle (Fig. 1).

\section{The Presence of Moisture in Refrigeration Cycle}

Another contaminant in the refrigeration system is moisture, which can get into the installation with oil.

Refrigeration systems are very sensitive to moisture in the refrigerant side of the system. If moisture gets into the system, failure may occur due to:

- ice formation in expansion valves, capillary tubes or evaporators;

- corrosion of metals;

- copper plating;

- chemical damage to insulation in hermetic compressors or other system materials;

Sources of moisture in the refrigeration system include:

- faulty equipment drying in factories and service operations;

- introduction of moisture during installation or service operations in the field;

- low-side leaks (resulting in entrance of moisture-laden air);

- leakage of water-cooled condenser;

- oxidation of certain hydrocarbons of oil to produce moisture;

- wet oil, refrigerant or both;

- decomposing cellulose insulation in hermetically sealed units.

Water freely absorbs into refrigerant as if soaked up by a dry sponge. Due to its solubility characteristics, particularly with HCFC and HFC refrigerants, water will continue to be absorbed into refrigerant until saturation is achieved. Once saturation is exceeded, a flooded condition is created (see Flooded Chiller) and the actual amount of water in the system cannot be determined by refrigerant analysis.

The impact of moisture in refrigerant can not be overstated. Refrigerant moisture directly causes the formation of acids resulting in metal corrosion, copper plating and chemical damage to the insulation in hermetic compressors and other system materials. Metal corrosion may lead to rust and pitting of motor bearings and copper plating may form on bearings causing a reduction in tolerance. Wet refrigerant may also lead to ice formation in expansion valve, capillary tubes or evaporators. Moisture contamination occurs when moisture enters the refrigeration system. Moisture can exist in three forms water, solid and gas. A compressor is designed to perform efficiently under specific controlled conditions.

Therefore, if contaminants are introduced into the system they will act to reduce compressor efficiency, effectiveness and durability. If moisture enters in to a refrigeration system, it combines with the refrigerant to form an acidic solution which may erode internal compressor components. Moisture does not actually cause direct compressor failure; the failure results from the failure of a part which has been weakened as a result of the effects of rust and/or corrosion.

\section{The Presence of Lubricant in Refrigeration Cycle}

A lubrication agent is necessary in almost all the vapor compression systems, particularly for the correct operation of the positive displacement compressor. The cylinder-Piston assembly, the reciprocating movement and friction of piston give the main characteristic of a positive displacement compressor. The main role of oil is to ensure the existence of a thin oil film allowing the lubrication of the mechanical moving elements (pistons, connecting $\mathrm{rod} / \mathrm{crank}$, valves, etc. to protect them against wear. 
To reduce the friction, the lubricant is used.

It assures a correct compressor operation and cylinder-Piston assembly performance. Because of shortage of data about the amount of oil circulating in the cycle, it is exemplified contained in ISO deal with the amount of oil in the compressor. Inside the lubricated compressor the refrigerant, unavoidably, picks up some mineral oil or synthetic lubricant. In addition, the refrigerant from the non-lubricated (dry) compressor may contain oil in the refrigerant (in Fig. 4).

Lubricant, according to ISO 8573, in compressed air can belong to one of the three following categories:

- bulk liquid;

- aerosol;

- vapour [2].

The amount of oil (concentration) in air is presented in ISO 8573 [5].

Although oil, moisture and air existed in refrigeration systems, they are not regarded in the thermodynamic calculation. In the first part of this article the thermodynamic calculation for a mixture consists of oil, moisture and air (oil and moist air). It could help to describe the mixture consisting of refrigerant with oil, moisture and air.

\section{The Mathematics Modeling of a Mixture of Moist Air and Lubricant in the Vertical Cylinder}

The humid air, in thermodynamics, is considered a mixture of ideal gases. However, such an approach is not entirely appropriate, when an influence of higher pressures and temperatures is taken into account in the compressor. The lubricating substances could be divided into natural mineral oils, synthetic oil and half-synthetic oil. The mineral oils could further be divided into: (1) with a small amount of aromatic hydrocarbons, known as paraffin oils; (2) oils with a majority of aromatic hydrocarbons, known as naphthalene oils; (3) oils with a majority of aromatic hydrocarbons supplemented by groups of simple or alkyl rings. All these oils were elementary lubrication
Table 1 Maximum oil content [3].

\begin{tabular}{ll}
\hline Class & Maximum concentration $\left(\mathrm{mg} / \mathrm{m}^{3}\right)$ \\
\hline 1 & 0.01 \\
2 & 0.1 \\
3 & 1 \\
4 & 5 \\
5 & 25 \\
\hline
\end{tabular}

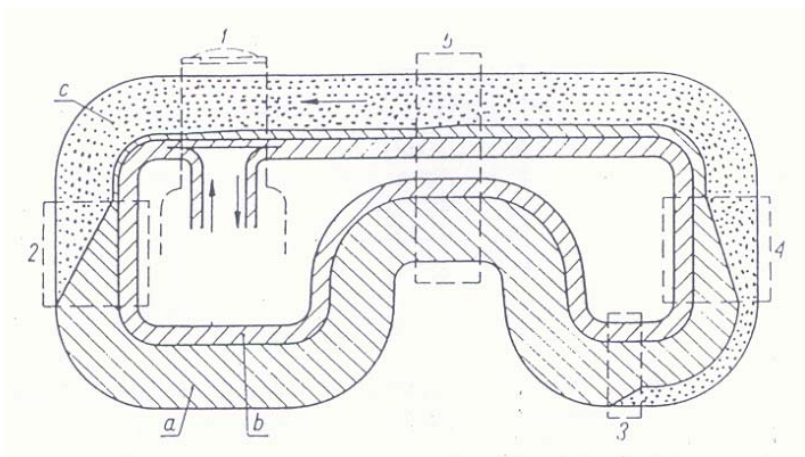

Fig. 4 Scheme of cycle of refrigerant and oil in refrigerant cycle: a-liquid oil; b-vapor oil; 1-compressor, 2-condenser; 3-throttling valve; 4-evaporator; 5-heat exchanger.

factors used in technology of air-compression and refrigeration. The progress of technique results in increasing number of lubrication substances being used. The wide scope of research carried out in this field resulted in creation of many new synthetic lubrication substances. In the cylinder of positive displacement air compressor where a lubricating substance is used as a sealing, cooling and lubricating, the compressor is actually compressing the mixture of humid air and lubricating substance [6].

During the lubricating of the cylinder's smooth surface, an oil-film is emerging on the cylinder's wall (in Fig. 6). Furthermore, a partial dispersion of lubricating substance takes place in the working space of the compressor that results in appearance of aerosol. As a consequence of the Piston's forward-backward movement and its friction against the cylinder's wall, the wall's temperature rises. If the temperature is high enough, the wall "dries out". The oil takes over the heat from the cylinder's wall (cooling function) and turns into vapour. In the compressor cylinder a structure of flux of the air and lubricating substance 


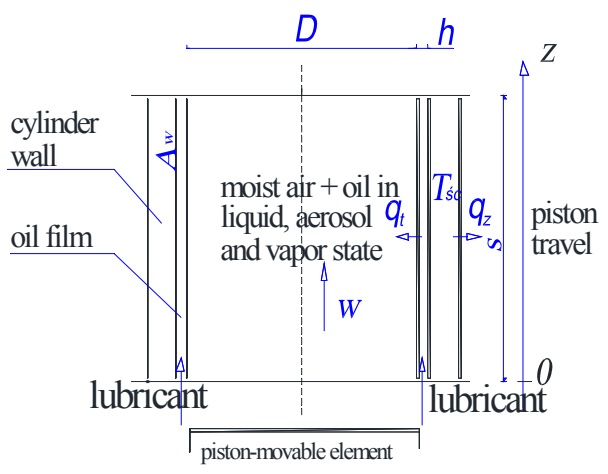

Fig. 5 Scheme of compression in piston of compressor [6].

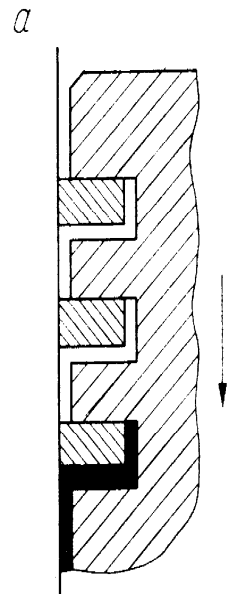

GMP

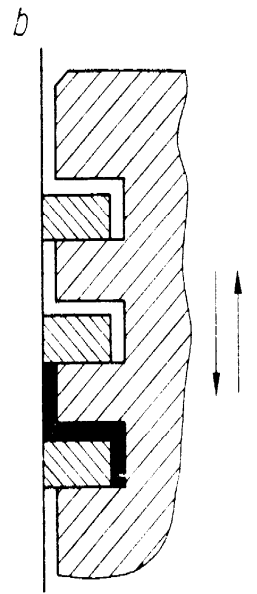

DMP

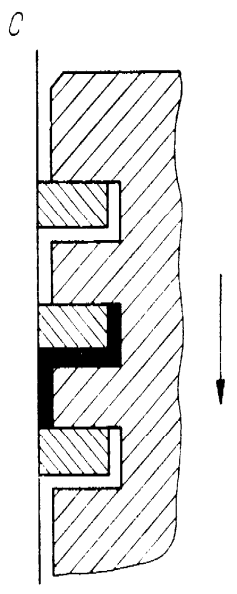

GMP
Fig. 6 Scheme blow up of operation of piston ring.

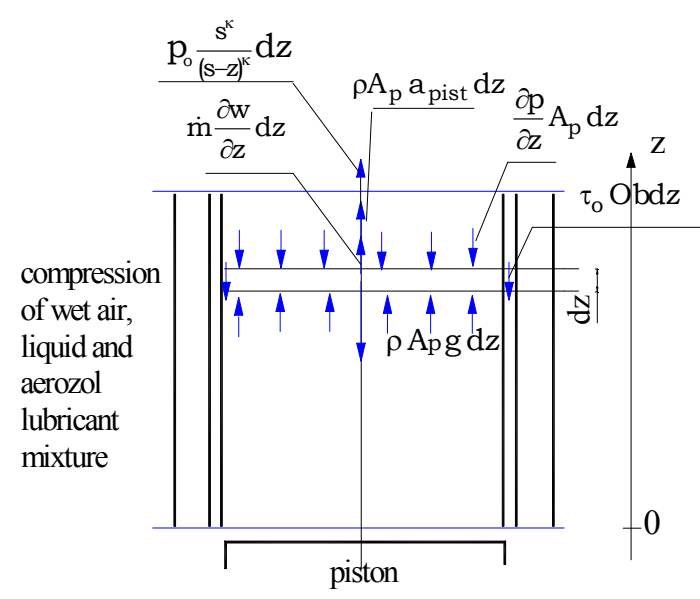

Fig. 7 Forces operative on elementary layer of fluid.

mixture in the form of liquid, steam and aerosol is emerging. The heat that is transferred during the exchange between the air and external environment is described as the external heat of transformation $\dot{q}_{z}$. (in Fig. 5) The value of this heat and its sign influence a pace of thermodynamic transformation taking place in the compressor directly. It is assumed in the paper that the process of compression is accompanied of exchange of the heat with the environment. Thus, in order to solve equation systems constituting a mathematics model of compression process what is needed is knowledge of a density of the stream of heat relating to the length of piston stroke.

\subsection{Mass Conservation, Momentum Conservation, Energy Conservation Low}

In the analyzed case of compression air-lubricant mixture we are dealing with the "haze" system. The phenomenological method has been adopted here to describe the model. This method assumes that it is justified to treat two-phase liquid as the two penetrating and interacting continuous units.

The two-phase as well as one-phase flux is governed by the mass, momentum conservation and energy conservation laws supplemented by constitutive equations (i.e., equations of state, strain, momentum transport and energy transport as well as chemical features of the liquid). The governing equations of the flow (continuity, momentum and energy) can be displayed either in the form of integral or differential (in Fig. 7).

The mass conservation law adapted to the flowing mixture is given by Eq. (1).

$$
A_{p}\left(\frac{\partial \rho}{\partial \tau}\right)+\frac{\partial \dot{m}}{\partial z}=0
$$

Table 2 Specific enthalpy of mixture.

\begin{tabular}{|c|c|c|c|}
\hline$T$ & $\begin{array}{l}\text { Specific } \\
\text { enthalpy of wet } \\
\left.\text { air }^{*}\right)\end{array}$ & $\begin{array}{l}\text { Specific } \\
\text { enthalpy } \\
\text { lubricant }\end{array}$ & $\begin{array}{l}\text { Specific } \\
\text { enthalpy } \\
\text { mixture }\end{array}$ \\
\hline K & $\mathrm{kJ} / \mathrm{kg}$ & $\mathrm{kJ} / \mathrm{kg}$ & $\mathrm{kJ} / \mathrm{kg}$ \\
\hline 298 & 809.26 & 46.15516394 & 809.7264089 \\
\hline 308 & 822.42 & 65.53847094 & 823.0778743 \\
\hline 318 & 835.55 & 85.45291269 & 836.4038873 \\
\hline 328 & 848.64 & 105.9199671 & 849.7028627 \\
\hline 338 & 861.76 & 126.9426286 & 863.0291637 \\
\hline 353 & 881.49 & 159.5379534 & 883.0813217 \\
\hline
\end{tabular}

" Example results of testing, when the pressure of compressed air is $0.2(\mathrm{MPa})$. 
Considering the force balance on an elementary layer of mixture in the flow field is given by Eq. (2):

$$
\frac{\partial \dot{m}}{\partial \tau}-A_{p} \frac{d p}{d z}=\tau_{0} * O b+A_{p} \rho \frac{w d w}{d z}-A_{p} \rho g+A_{p} \rho a_{p i s t}+p_{o} \frac{s^{K}}{(s-z)^{K}}
$$

The energy conservation law gives:

$$
\rho A_{p} \frac{\partial h}{\partial \tau}+\frac{d\left[\dot{m}\left(h+\frac{w^{2}}{2}\right)\right]}{d z}=\pi D_{i n} \dot{q}_{z}-A_{p} \rho g w
$$

\subsection{A Method of the Solution of an Example}

The presented mathematical model for the compression process takes a form of differential equations, depicting a change of the selected parameters along the cylinder's length, in the form that is directly useful for computer programs:

The initial values used for mathematics models solution is: (1) initial air pressure equal atmospheric pressure; (2) initial specific enthalpy value of the air in the compressors' entrance.

The presented system of differential equations can be solved by numeric integration methods. The Runge-Kutty fifth order method has been adopted in order to solve the above-mentioned differential equations [6].

\section{Conclusions}

This article presents an analysis of research directions related to thermodynamic aspects of the presence of contaminants in refrigerant. The author would like to underline that contaminants like oil, air, water vapor exist in refrigeration cycle but are not taken account in calculations. In this paper, the author describes mixture consisting of moist air and lubricant by means of differential equations mass conservation law, momentum conservation and energy conservation law. Exemplary calculations show differences between specific enthalpy of dry air, wet air and mixture of wet air and lubricant in liquid and aerosol state in the elevated pressure and temperature (Table 2).

\section{References}

[1] Y. Hwang, R. Radermacher, T. Hirata, Oil mass fraction measurement of $\mathrm{CO}_{2} / \mathrm{PAG}$ mixture, International Journal of Refrigeration 31 (2) (2008) 256-261.

[2] M. Youbi-Idrissi, J. Bonjour, The effect of oil refrigeration, current research issues and critical review of thermodynamic aspects, International Journal of Refrigeration 31 (2) (2008) 165-179.

[3] K.W. Cooper, A.G. Mount, Oil circulation-Its effect on compressor capacity, theory and experiment, in: Proceedings of the 1974 Purdue Compressor Technology Conference, 1974.

[4] D. Butrymowicz, An Impact inert gases and overheating of vapor on work of condenser, Technika chłodnicza I klimatyzacyjna, 2003.

[5] ISO 8573-1, Compressed Air for General Use, 1991.

[6] D. Skrzyniowska, A model of air and lubricant mixture from a possitive displacement compressor, Dr. Thesis, Cracow University of Technology, 2000. 